\title{
Schultz and Modified Schultz Polynomials for Vertex - Identification Chain and Ring - for Hexagon Graphs
}

\author{
Mahmood M. Abdullah \\ Ahmed M. Ali \\ mmadain7@gmail.com \\ ahmedgraph@uomosul.edu.iq \\ Department of Mathematics, \\ College of Computer Science and Mathematics, \\ University of Mosul, Mosul, Iraq
}

Received on: 09/04/2020

Accepted on: 07/05/2020

\section{ABSTRACT}

The aim of this paper is to find polynomials related to Schultz, and modified Schultz indices of vertex identification chain and ring for hexagonal rings $(6-$ cycles). Also to find index and average index of all of them.

Keywords: Schultz, modified Schultz, vertex identification chain and ring.

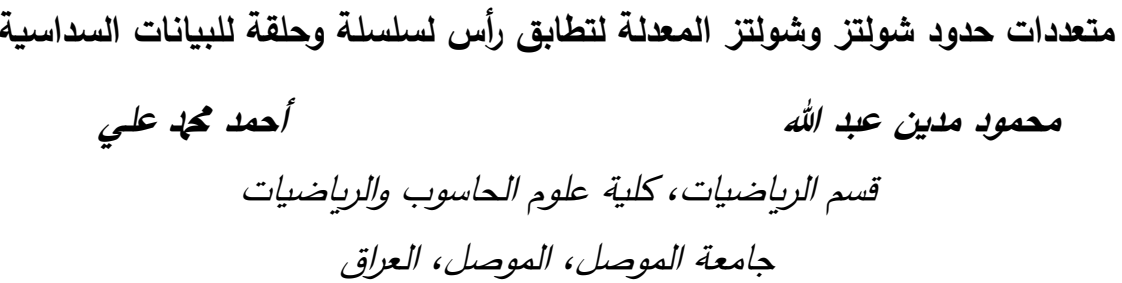

تاريخ قبول البحث: 2020/05/07

تاريخ استلام البحث: 2020/04/09

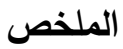

الهدف من هذا البحث هو ايجاد متعددات حدود شولتز وشولتز المعدلة لتطابق رأس لسلسلة وحلقة للحلقات
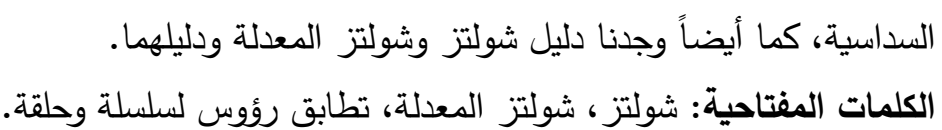

\section{INTRODUCTION:}

We will let all graphs in this paper to be connected, finite, undirected and simple, which means empty from loops and multiple edges. Let $G=(V, E)$ be a connected simple graph, and $V=V(G)$ and $E=E(G)$ denote the sets of vertices and edges, respectively, of $G$.

In any graph $G$ represent the number of vertices the order of $G$ and denoted that by symbol $p=p(G)=|V(G)|$, and we called the number of edges the size of $G$, and denoted that by symbol $q=q(G)=|E(G)|$. We say for any two vertices $u, v$ in $G$ adjacent in $G$ if there exists edge between them, and we write $e=u v$, as well as we say the edge $e$ incident on $u$ and $v$. We called the degree of vertex $u$ as the number of edges incident on it and denoted that by degu as such that for vertex $v$ in $G$ [5].

Now, we define the distance between any two vertices $u, v$ in $G$. The distance is the length of a shortest path that join between $u$ and $v$ in $G$ which is denoted by $d_{G}(u, v)$ or $d(u, v)$. We called the maximum distance between any two vertices $u$ and $v$ in $G$ the diameter and denoted that by $\operatorname{diam} G$ [4]. In 2005, Gutman introduced the graph 
polynomials related to the Schultz and modified Schultz indices [12], and in 2011, Behmaram, et al. found the Schultz polynomials of some graph operation [3]. Farahani [9], gave Schultz and modified Schultz polynomials of some Harary graphs in 2013. Ahmed and Haitham studied Schultz and modified Schultz polynomials, indices, and index average for two Gutman's operations [1]. Also they found general formulas for Schultz and modified Schultz polynomials, indices, and index average of cog-special graphs [2]. Also there are many studies about their applications $([6,7,8,10,11])$.

Schultz had introduced and studied in 1989 Schultz index (molecular topological index) [18]. Then, in 1997 Klavžar and Gutman introduced the modified Schultz index [17].

They have defined Schultz and modified Schultz, indices, respectively, as:

$S c(G)=\sum_{\{u, v\} \subseteq V(G)}(\operatorname{deg} v+\operatorname{deg} u) d(u, v)$.

$S c^{*}(G)=\sum_{\{u, v\} \subseteq V(G)}(\operatorname{deg} v \cdot \operatorname{deg} u) d(u, v)$.

Schultz and modified Schultz polynomials are considered very important polynomials through studying some properties of their coefficients. Schultz and modified Schultz polynomials are defined, respectively, as:

$\operatorname{Sc}(G ; x)=\sum_{\{u, v\} \subseteq V(G)}(\operatorname{deg} v+\operatorname{deg} u) x^{d(u, v)}$.

$S c^{*}(G ; x)=\sum_{\{u, v\} \subseteq V(G)}(\operatorname{deg} v \cdot \operatorname{degu}) x^{d(u, v)}$.

We can obtain the indices of Schultz and modified Schultz by taking derivative of them with respect to $x$ at $x=1$, as explained below.

$$
S c(G)=\left.\frac{d}{d x}(S c(G ; x))\right|_{x=1} \text { and } S c^{*}(G)=\left.\frac{d}{d x}\left(S c^{*}(G ; x)\right)\right|_{x=1} \text {. }
$$

While we can obtain the average of the Schultz and modified Schultz indices for connected graph $G$ with order $p(G)$ that are defined as:

$\overline{S c}(G)=2 S c(G) / p(G)(p(G)-1)$ and $\overline{S c^{*}}(G)=2 S c^{*}(G) / p(G)(p(G)-1)$.

In any connected graph $G$, we refer to the set of unordered pairs of vertices which are distance $k$ apart by the symbol $D_{k}(G)$ and let $\left|D_{k}(G)\right|=D(G, k)$.

Now let that $D_{k}(r, h)$ be the set of all unordered pairs of vertices $u, v$ in $G$, which are of distance $k$ and of $\operatorname{deg} u=r, \operatorname{deg} v=h$.

It is obvious that $\sum_{k=1}^{\operatorname{diam}(G)}\left|D_{k}(G)\right|=p(G)(p(G)-1) / 2$, where $D(G, k)=$ $\left|D_{k}(G)\right|$.

Finally, Schultz indices are considered very interesting to determine some properties of chemical structures, see more $([13,14,15,16])$.

\section{Main Results:}

\subsection{The Vertex - Identification Chain (VIC) - Graphs:}

Let $\left\{G_{1}, G_{2}, \ldots, G_{n}\right\}$ be a set of pairwise disjoint graphs with vertices $u_{i}, v_{i} \in V\left(G_{i}\right), i=1,2, \ldots, n, n \geq 2$, then the vertex-identification chain graph $C_{v}\left(G_{1}, G_{2}, \ldots, G_{n}\right) \equiv C_{v}\left(G_{1}, G_{2}, \ldots, G_{n}: v_{1} \cdot u_{2} ; v_{2} \cdot u_{3} ; \ldots ; v_{n-1} \cdot u_{n}\right)$ of $\left\{G_{i}\right\}_{i=1}^{n}$ with respect to the vertices $\left\{v_{i}, u_{i+1}\right\}_{i=1}^{n-1}$ is the graph obtained from the graphs $G_{1}, G_{2}, \ldots, G_{n}$ by identifying the vertex $v_{i}$ with the vertex $u_{i+1}$ for all $i=1,2, \ldots, n-1$. (See Fig. 2-1) in which: 

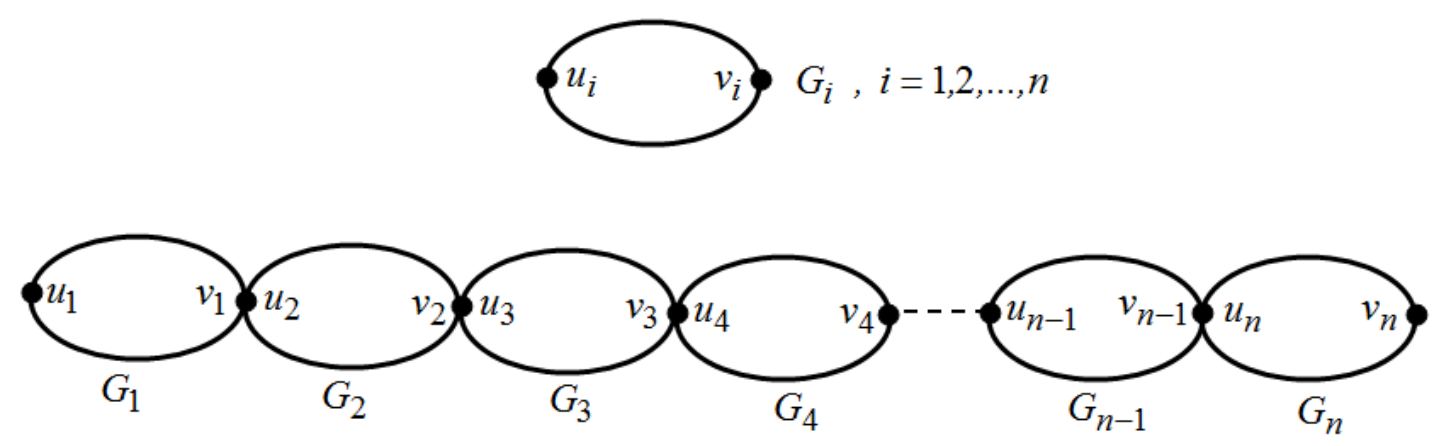

Fig. 2-1-1. $C_{v}\left(G_{1}, G_{2}, \ldots, G_{n}\right)$.

Some Properties of Graph $C_{v}\left(G_{1}, G_{2}, \ldots, G_{n}\right)$ :

1. $p\left(C_{v}\left(G_{1}, G_{2}, \ldots, G_{n}\right)\right)=\sum_{i=1}^{n} p\left(G_{i}\right)-(n-1)$.

2. $q\left(C_{v}\left(G_{1}, G_{2}, \ldots, G_{n}\right)\right)=\sum_{i=1}^{n} q\left(G_{i}\right)$.

3. $n \leq \operatorname{diam}\left(C_{v}\left(G_{1}, G_{2}, \ldots, G_{n}\right)\right) \leq \sum_{i=1}^{n} \operatorname{diam}\left(G_{i}\right)$.

The equality of both bounds are satisfied at complete graphs, but the upper bound is satisfied at path graphs in which $v_{i}, u_{i}$ are end-vertices of $G_{i}$ for $i=1,2, \ldots, n$.

If $G_{i} \equiv H_{p}$, for all $1 \leq i \leq n$, where $H_{p}$ is a connected graph of order $p$, we denoted $C_{v}\left(H_{p}, H_{p}, \ldots, H_{p}\right)$ by $C_{v}\left(H_{p}\right)_{n}$.

Schultz and modified Schultz of $\boldsymbol{C}_{v}\left(C_{6}\right)_{p / 2}$

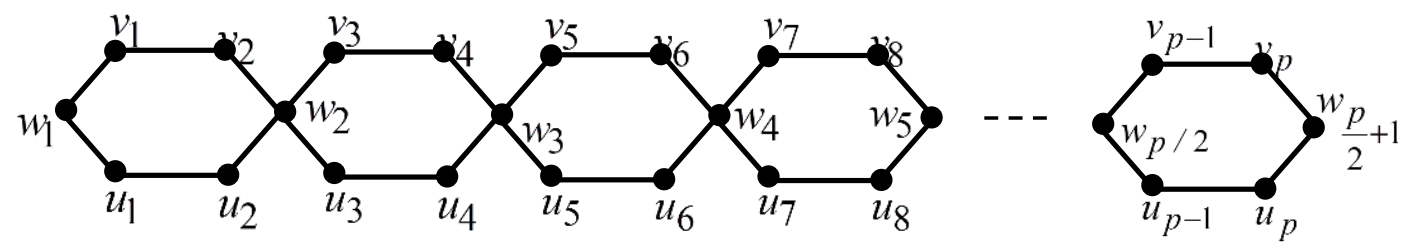

Fig. 2-1-2 The Graph $C_{v}\left(C_{6}\right)_{p / 2}$

From Fig. 2-1-2, we note that $p\left(C_{v}\left(C_{6}\right) \frac{p}{2}\right)=\frac{5 p}{2}+1, q\left(C_{v}\left(C_{6}\right) \frac{p}{2}\right)=3 p$ and $\operatorname{diam}\left(C_{v}\left(C_{6}\right) \frac{p}{2}\right)=\frac{3 p}{2}$. For all $1 \leq i, j \leq p, i \neq j$ and $2 \leq m, h \leq \frac{p}{2}, m \neq h$ we have:

Table 2.1

\begin{tabular}{|c|c|c|c|c|c|}
\hline$x$ & $\operatorname{deg} u_{i}=2$ & $\operatorname{deg} v_{i}=2$ & $\operatorname{deg} w_{1}=2$ & $\begin{array}{l}\operatorname{deg} w \frac{p}{2}+1 \\
=2\end{array}$ & $\operatorname{deg} w_{m}=4$ \\
\hline $\operatorname{deg} u_{j}=2$ & & & & & \\
\hline $\operatorname{deg} v_{j}=2$ & & & & & \\
\hline $\operatorname{deg} w_{1}=2$ & & & & $\begin{array}{l}4 \\
4 \\
\end{array}$ & \\
\hline $\begin{array}{l}\operatorname{deg} w \frac{p}{2}+1 \\
=2\end{array}$ & & & & & \\
\hline $\operatorname{deg} w_{h}=4$ & 8 & $\frac{6}{8}$ & $\frac{6}{8}$ & $\frac{6}{8}$ & \\
\hline
\end{tabular}


Theorem 2.1.1: For $p \geq 4$, then:

$$
\begin{aligned}
& \text { 1. } S c\left(C_{v}\left(C_{6}\right) \frac{p}{2} ; x\right)=8(2 p-1) x+24(p-1) x^{2}+12(2 p-3) x^{3} \\
& +\frac{20}{3} \sum_{k=4}^{\frac{3 p}{2}}(3 p-2 k) x^{k}+\frac{4}{3} x\left(3 x^{2}+2 x+4\right) \sum_{k=1}^{\frac{p}{2}-1} x^{3 k} . \\
& \text { 2. } S c^{*}\left(C_{v}\left(C_{6}\right) \frac{p}{2} ; x\right)=4(5 p-4) x+4(7 p-8) x^{2}+4(7 p-12) x^{3} \\
& +\sum_{k=4}^{\frac{3 p}{2}-1}(24 p-16) x^{k}+4 x^{\frac{3 p}{2}} .
\end{aligned}
$$

Proof: For all $p \geq 8$ and every two vertices $u, v \in V\left(C_{v}\left(C_{6}\right) \frac{p}{2}\right)$, there is $d(u, v)=k$, $1 \leq k \leq \frac{3 p}{2}$, we will have ten partitions for proof:

P1. If $d(u, v)=1$, then $\left|D_{1}\right|=3 p=q\left(C_{v}\left(C_{6}\right) \frac{p}{2}\right)$ and we have two subsets of the edge set:

$$
\begin{aligned}
\text { P1.1 }\left|D_{1}(2,2)\right|=\mid & \left\{\left(u_{2 i-1}, u_{2 i}\right),\left(v_{2 i-1}, v_{2 i}\right): 1 \leq i \leq \frac{p}{2}\right\} \cup\left\{\left(w_{1}, u_{1}\right),\left(w_{1}, v_{1}\right),\left(w_{\frac{p}{2}+1}, u_{p}\right),\right. \\
& \left.\left(w_{\frac{p}{2}+1}, v_{p}\right)\right\} \mid=p+4 .
\end{aligned}
$$

P1.2 $\left|D_{1}(2,4)\right|=\left|\left\{\left(u_{2 i}, w_{i+1}\right),\left(v_{2 i}, w_{i+1}\right),\left(u_{2 i+1}, w_{i+1}\right),\left(v_{2 i+1}, w_{i+1}\right): 1 \leq i \leq \frac{p}{2}-1\right\}\right|$

$$
=2 p-4 \text {. }
$$

P2. If $d(u, v)=2$, then, we have two subsets of $D_{2}$

P2.1 $\left|D_{2}(2,2)\right|=\mid\left\{\left(u_{2 i}, u_{2 i+1}\right),\left(v_{2 i}, v_{2 i+1}\right),\left(u_{2 i}, v_{2 i+1}\right),\left(v_{2 i}, u_{2 i+1}\right): 1 \leq i \leq \frac{p}{2}-1\right\} \cup$

$$
\begin{aligned}
& \left\{\left(w_{1}, u_{2}\right),\left(w_{1}, v_{2}\right),\left(w_{\frac{p}{2}+1}, u_{p-1}\right),\left(w_{\frac{p}{2}+1}, v_{p-1}\right)\right\} \cup\left\{\left(u_{i}, v_{i}\right): 1 \leq i \leq p\right\} \mid \\
& =3 p .
\end{aligned}
$$

P2.2 $\left|D_{2}(2,4)\right|=\mid\left\{\left(u_{2 i-1}, w_{i+1}\right),\left(v_{2 i-1}, w_{i+1}\right),\left(u_{2 i+2}, w_{i+1}\right),\left(v_{2 i+2}, w_{i+1}\right)\right.$ :

$$
\left.1 \leq i \leq \frac{p}{2}-1\right\} \mid=2 p-4 \text {. }
$$

Therefor $\left|D_{2}\right|=5 p-4$.

P3. If $d(u, v)=3$, then, we have three subsets of $D_{3}$ :

P3.1 $\left|D_{3}(2,2)\right|=\mid\left\{\left(u_{i}, u_{i+2}\right),\left(v_{i}, v_{i+2}\right),\left(u_{i}, v_{i+2}\right),\left(v_{i}, u_{i+2}\right): 1 \leq i \leq p-2\right\} \cup$

$$
\left\{\left(u_{2 i-1}, v_{2 i}\right),\left(v_{2 i-1}, u_{2 i}\right): 1 \leq i \leq \frac{p}{2}\right\} \mid=5 p-8 \text {. }
$$

P3.2 $\left|D_{3}(2,4)\right|=\left|\left\{\left(w_{1}, w_{2}\right),\left(w_{\frac{p}{2}+1}, w \frac{p}{2}\right)\right\}\right|=2$.

P3.3 $\left|D_{3}(4,4)\right|=\left|\left\{\left(w_{i+1}, w_{i+2}\right): 1 \leq i \leq \frac{p}{2}-2\right\}\right|=\frac{p}{2}-2$.

Therefor $\left|D_{3}\right|=\frac{11 p}{2}-8$.

P4. If $d(u, v)=k$, when $k=3 j+4, \mathrm{j}=0,1, \ldots, \frac{p}{2}-3$, then, we have two subsets of $D_{k}$ :

$$
\begin{aligned}
& \text { P4.1 }\left|D_{k}(2,2)\right|=\mid\left\{\left(u_{2 i-1}, u_{2 i+\frac{2(k-1)}{3}}\right),\left(v_{2 i-1}, v_{2 i+\frac{2(k-1)}{3}}\right),\left(u_{2 i-1}, v_{2 i+\frac{2(k-1)}{3}}\right)\right. \text {, } \\
& \left.\left(v_{2 i-1}, u_{2 i+\frac{2(k-1)}{3}}\right): 1 \leq i \leq \frac{p}{2}-\frac{k-1}{3}\right\} \cup\left\{\left(w_{1}, \frac{u_{2 k+1}}{3}\right),\left(w_{1}, \frac{v_{2 k+1}}{3}\right)\right. \text {, } \\
& \left.\left(w_{\frac{p}{2}+1}, u_{p-\frac{2(k-1)}{3}}\right),\left(w_{\frac{p}{2}+1}, v_{p-\frac{2(k-1)}{3}}\right)\right\} \mid=2 p-\frac{4(k-4)}{3} \text {. } \\
& \text { P4.2 }\left|D_{k}(2,4)\right|=\mid\left\{\left(u_{2 i}, w_{i+\frac{k+2}{3}}\right),\left(v_{2 i}, w_{i+\frac{k+2}{3}}\right),\left(u_{2 i+\frac{2 k+1}{3}}, w_{i+1}\right),\left(v_{2 i+\frac{2 k+1}{3}}, w_{i+1}\right)\right. \text { : } \\
& \left.1 \leq i \leq \frac{p}{2}-\frac{k+2}{3}\right\} \mid=2 p-\frac{4(k+2)}{3} \text {. }
\end{aligned}
$$


Therefore $\left|D_{k}\right|=4 p-\frac{8}{3}(k-1)$, for $k=3 j+4, \mathrm{j}=0,1, \ldots, \frac{p}{2}-3$

P5. If $d(u, v)=k$, when $k=3 j+5, \mathrm{j}=0,1, \ldots, \frac{p}{2}-3$, then, we have two subset of $D_{k}$ :

P5.1 $\left|D_{k}(2,2)\right|=\mid\left\{\left(u_{2 i}, u_{2 i+\frac{2 k-1}{3}}\right),\left(v_{2 i}, v_{2 i+\frac{2 k-1}{3}}\right),\left(u_{2 i}, v_{2 i+\frac{2 k-1}{3}}\right),\left(v_{2 i}, u_{2 i+\frac{2 k-1}{3}}\right)\right.$ :

$$
\begin{aligned}
& \left.1 \leq i \leq \frac{p}{2}-\frac{k+1}{3}\right\} \cup\left\{\left(w_{1}, u_{\frac{2(k+1)}{3}}\right),\left(w_{1}, \frac{\left.v_{\frac{2(k+1)}{3}}\right)}{3},\left(w_{\frac{p}{2}+1}, u_{p-\frac{2 k-1}{3}}\right),\right.\right. \\
& \left.\left(w_{\frac{p}{2}+1}, v_{p-\frac{2 k-1}{3}}\right)\right\} \mid=2 p-\frac{4(k-2)}{3} .
\end{aligned}
$$

P5.2 $\left|D_{k}(2,4)\right|=\mid\left\{\left(u_{2 i-1}, w_{i+\frac{k+1}{3}}\right),\left(v_{2 i-1}, w_{i+\frac{k+1}{3}}\right),\left(u_{2 i+\frac{2(k+1)}{3}}, w_{i+1}\right),\left(v_{2 i+\frac{2(k+1)}{3}}, w_{i+1}\right)\right.$

$$
\left.: 1 \leq i \leq \frac{p}{2}-\frac{k+1}{3}\right\} \mid=2 p-\frac{4(k+1)}{3} \text {. }
$$

Thus $\left|D_{k}\right|=4 p-\frac{4}{3}(2 k-1)$ for $k=3 j+5, \mathrm{j}=0,1, \ldots, \frac{p}{2}-3$.

P6. If $d(u, v)=k$, when $k=3 j+6, j=0,1, \ldots, \frac{p}{2}-4$, then, we have three subsets of $D_{k}$ :

P6.1 $\left|D_{k}(2,2)\right|=\mid\left\{\left(u_{i}, u_{i+\frac{2 k}{3}}\right),\left(v_{i}, v_{i+\frac{2 k}{3}}\right),\left(u_{i}, v_{i+\frac{2 k}{3}}\right),\left(v_{i}, u_{i+\frac{2 k}{3}}\right)\right.$ :

$$
\left.1 \leq i \leq p-\frac{2 k}{3}\right\} \mid=4 p-\frac{8 k}{3} \text {. }
$$

P6.2 $\left|D_{k}(2,4)\right|=\left|\left\{\left(w_{1}, w_{\frac{k}{3}+1}\right),\left(w_{\frac{p}{2}+1}, w_{\frac{p}{2}}-\frac{k}{3}+1\right)\right\}\right|=2$.

P6.3 $\left|D_{k}(4,4)\right|=\left|\left\{\left(w_{i+1}, w_{\mathrm{i}+\frac{k}{3}+1}\right): 1 \leq i \leq \frac{p}{2}-\frac{k}{3}-1\right\}\right|=\frac{p}{2}-\frac{k}{3}-1$.

Thus $\left|D_{k}\right|=\frac{9 p}{2}-3 k+1$ for $k=3 j+6, j=0,1, \ldots, \frac{p}{2}-4$.

P7. If $d(u, v)=\frac{3 p}{2}-3$, then, we have two subsets of $D_{\frac{3 p}{2}-3}$ :

P7.1 $\left|D_{\frac{3 p}{2}-3}(2,2)\right|=\left|\left\{\left(u_{i}, u_{p+i-2}\right),\left(v_{i}, v_{p+i-2}\right),\left(u_{i}, v_{p+i-2}\right),\left(v_{i}, u_{p+i-2}\right): i=1,2\right\}\right|$ $=8$.

P7.2 $\left|D_{\frac{3 p}{2}-3}(2,4)\right|=\left|\left\{\left(w_{1}, w \frac{p}{2}\right),\left(w_{\frac{p}{2}+1}, w_{2}\right)\right\}\right|=2$.

Therefore $\left|D_{\frac{3 p}{2}-3}\right|=10$.

P8. If $d(u, v)=\frac{3 p}{2}-2$, then $\left|D_{\frac{3 p}{2}-2}\right|=8$, because:

$\left|D_{\frac{3 p}{2}-2}(2,2)\right|=\mid\left\{\left(u_{1}, u_{p}\right),\left(u_{1}, v_{p}\right),\left(v_{1}, v_{p}\right),\left(v_{p}, u_{1}\right),\left(u_{2}, w \frac{p}{2}+1\right),\left(u_{p-1}, w_{1}\right)\right.$, $\left.\left(v_{2}, w \frac{p}{2}+1\right),\left(v_{p-1}, w_{1}\right)\right\} \mid=8$.

P9. If $d(u, v)=\frac{3 p}{2}-1$, then $\left|D_{\frac{3 p}{2}-1}\right|=4$, because:

$\left|D_{\frac{3 p}{2}-1}(2,2)\right|=\left|\left\{\left(u_{1}, w \frac{p}{2}+1\right),\left(u_{p}, w_{1}\right),\left(v_{1}, w \frac{p}{2}+1\right),\left(v_{p}, w_{1}\right)\right\}\right|=4$.

P10. If $d(u, v)=\frac{3 p}{2}$, then $\left|D_{\frac{3 p}{2}}\right|=1$, since $\left|D_{\frac{3 p}{2}}(2,2)\right|=\left|\left\{\left(w_{1}, w \frac{p}{2}+1\right)\right\}\right|=1$.

From $\mathrm{P}_{1}$ to $\mathrm{P}_{10}$ and Table 2.1.1, we have:

$$
\begin{aligned}
S c\left(C_{e}\left(C_{6}\right) \frac{p}{2} ; x\right) & =\{4(p+4)+6(2 p-4)\} x+\{4(3 p)+6(2 p-4)\} x^{2} \\
& +\left\{4(5 p-8)+6(2)+8\left(\frac{p}{2}-2\right)\right\} x^{3}
\end{aligned}
$$




$$
\begin{aligned}
& +\sum_{k=4,7,10, \ldots}^{\frac{3 p}{2}-5}\left\{4\left(2 p-\frac{4(k-4)}{3}\right)+6\left(2 p-\frac{4(k+2)}{3}\right)\right\} x^{k} \\
& +\sum_{k=5,8,11, \ldots}^{\frac{3 p}{2}-.}\left\{4\left(2 p-\frac{4(k-2)}{3}\right)+6\left(2 p-\frac{4(k+1)}{3}\right)\right\} x^{k} \\
& +\sum_{k=6,9,12, \ldots}^{\frac{3 p}{2}-6}\left\{4\left(4 p-\frac{8 k}{3}\right)+6(2)+8\left(\frac{p}{2}-\frac{k}{3}-1\right)\right\} x^{k} \\
& +\{4(8)+6(2)\} x^{\frac{3 p}{2}-3}+\{4(8)\} x^{\frac{3 p}{2}-2}+\{4(4)\} x^{\frac{3 p}{2}-1}+\{4(1)\} x^{\frac{3 p}{2}} . \\
& =8(2 p-1) x+24(p-1) x^{2}+12(2 p-3) x^{3} \\
& +4 \sum_{k=4,7,10, \ldots}^{\frac{3 p}{2}-2}\left(5 p-\frac{2(5 k-2)}{3}\right) x^{k}+4 \sum_{k=5,8,11, \ldots}^{\frac{3 p}{2}-1}\left(5 p-\frac{2(5 k-1)}{3}\right) x^{k} \\
& +4 \sum_{k=6,9,12, \ldots}^{\frac{3 p}{2}}\left(5 p-\frac{10 k-3}{3}\right) x^{k} . \\
& =8(2 p-1) x+24(p-1) x^{2}+12(2 p-3) x^{3} \\
& +\frac{20}{3} \sum_{k=4}^{\frac{3 p}{2}}(3 p-2 k) x^{k}+\frac{4}{3} x\left(3 x^{2}+2 x+4\right) \sum_{k=1}^{\frac{p}{2}-1} x^{k} .
\end{aligned}
$$

Now, we find modified Shultz polynomial:

$$
\begin{aligned}
S c^{*}\left(C_{v}\left(C_{6}\right) \frac{p}{2} ; x\right) & =\{4(p+4)+8(2 p-4)\} x+\{4(3 p)+8(2 p-4)\} x^{2} \\
& +\left\{4(5 p-8)+8(2)+16\left(\frac{p}{2}-2\right)\right\} x^{3} \\
& +\sum_{k=4,7,10, \ldots}^{\frac{3 p}{2}-5}\left\{4\left(2 p-\frac{4(k-4)}{3}\right)+8\left(2 p-\frac{4(k+2)}{3}\right)\right\} x^{k} \\
& +\sum_{k=5,8,11, \ldots}^{\frac{3 p}{2}-4}\left\{4\left(2 p-\frac{4(k-2)}{3}\right)+8\left(2 p-\frac{4(k+1)}{3}\right)\right\} x^{k} \\
& +\sum_{k=6,9,12, \ldots}^{\frac{3 p}{2}-6}\left\{4\left(4 p-\frac{8 k}{3}\right)+8(2)+16\left(\frac{p}{2}-\frac{k}{3}-1\right)\right\} x^{k} \\
& +\{4(8)+8(2)\} x^{\frac{3 p}{2}-3}+\{4(8)\} x^{\frac{3 p}{2}-2}+\{4(4)\} x^{\frac{3 p}{2}-1}+\{4(1)\} x^{\frac{3 p}{2}} . \\
& =4(5 p-4) x+4(7 p-8) x^{2}+4(7 p-12) x^{3} \\
& +\sum_{k=4,7,10, \ldots}^{\frac{3 p}{2}-2}(24 p-16 k) x^{k}+\sum_{k=5,8,11, \ldots}^{\frac{3 p}{2}-1}(24 p-16 k) x^{k} \\
& +\sum_{k=6,9,12, \ldots}^{\frac{3 p}{2}-3}(24 p-16 k) x^{k}+4 x^{\frac{3 p}{2}} . \\
& =4(5 p-4) x+4(7 p-8) x^{2}+4(7 p-12) x^{3} \\
& +\sum_{k=4}^{\frac{3 p}{2}-1}(24 p-16 k) x^{k}+4 x^{\frac{3 p}{2}} .
\end{aligned}
$$

\section{Remark:}

1. $S c\left(C_{v}\left(C_{6}\right)_{2} ; x\right)=56 x+72 x^{2}+60 x^{3}+32 x^{4}+16 x^{5}+4 x^{6}$. $S c^{*}\left(C_{v}\left(C_{6}\right)_{2} ; x\right)=64 x+80 x^{2}+64 x^{3}+32 x^{4}+16 x^{5}+4 x^{6}$.

2. $S c\left(C_{v}\left(C_{6}\right)_{3} ; x\right)=88 x+120 x^{2}+108 x^{3}+72 x^{4}+56 x^{5}+44 x^{6}+32 x^{7}+16 x^{8}$

$$
+4 x^{9} \text {. }
$$

3. $S c^{*}\left(C_{v}\left(C_{6}\right)_{3} ; x\right)=104 x+136 x^{2}+120 x^{3}+80 x^{4}+64 x^{5}+48 x^{6}+32 x^{7}+16 x^{8}$ $+4 x^{9}$.

Corollary 2.1.2: For $p \geq 4$, then we have:

1. $S c\left(C_{v}\left(C_{6}\right)_{p / 2}\right)=\frac{3 p}{2}\left(5 p^{2}+3 p+10\right)$.

2. $S c^{*}\left(C_{v}\left(C_{6}\right)_{p / 2}\right)=9 p\left(p^{2}+2\right)$.

Corollary 2.1.3: If $n$ is the number of cycles $C_{6}$ in the graph $C_{v}\left(C_{6}\right)_{n}, n \geq 2$, then

1. $S c\left(C_{v}\left(C_{6}\right)_{n}\right)=6 n\left(10 n^{2}+3 n+5\right)$. 
2. $S c^{*}\left(C_{v}\left(C_{6}\right)_{n}\right)=36 n\left(2 n^{2}+1\right)$

Corollary 2.1.4: For $p \geq 4$, then we have:

1. $\overline{S c}\left(C_{v}\left(C_{6}\right) \frac{p}{2}\right)=\frac{12}{25}\left(5 p+1+\frac{48}{5 p+2}\right)$.

2. $\overline{S c^{*}}\left(C_{v}\left(C_{6}\right)_{p-1}\right)=\frac{72}{125}\left(5 p-2+\frac{54}{5 p+2}\right)$.

\subsection{The Vertex - Identification Ring (VIR) - Graph:}

Let $\left\{G_{1}, G_{2}, \ldots, G_{n}\right\}$ be a set of pairwise disjoint graphs with vertices $u_{i}, v_{i} \in V\left(G_{i}\right), i=1,2, \ldots, n, \geq 3$, then the vertex-identification Ring graph $R_{v}\left(G_{1}, G_{2}, \ldots, G_{n}\right) \equiv R_{v}\left(G_{1}, G_{2}, \ldots, G_{n}: v_{1} \cdot u_{2} ; v_{2} \cdot u_{3} ; \ldots ; v_{n-1} \cdot u_{n} ; v_{n} \cdot u_{1}\right)$ of $\left\{G_{i}\right\}_{i=1}^{n}$ with respect to the vertices $\left\{v_{i}, u_{i}\right\}_{i=1}^{n}$ is the graph obtained from the graphs $G_{1}, G_{2}, \ldots, G_{n}$ by identifying the vertex $v_{i}$ with the vertex $u_{i+1}$ for all $i=1,2, \ldots, n$. (See Fig. 2-2) where $u_{n+1} \equiv u_{1}$.

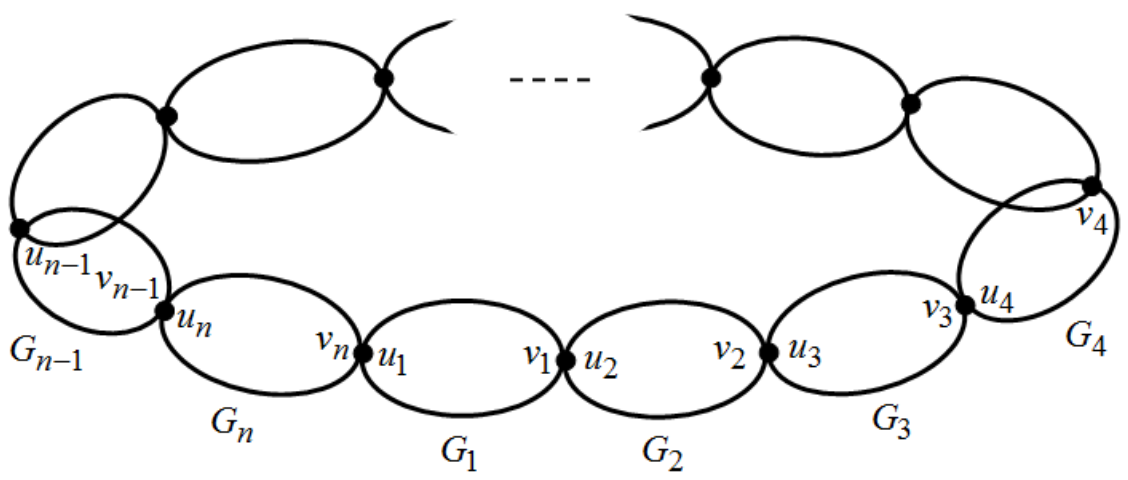

Fig. 2-2-1. $R_{v}\left(G_{1}, G_{2}, \ldots, G_{n}\right)$

Some Properties of the graph $R_{v}\left(G_{1}, G_{2}, \ldots, G_{n}\right)$ :

1. $p\left(R_{v}\left(G_{1}, G_{2}, \ldots, G_{n}\right)\right)=\sum_{i=1}^{n} p\left(G_{i}\right)-n$.

2. $q\left(R_{v}\left(G_{1}, G_{2}, \ldots, G_{n}\right)\right)=\sum_{i=1}^{n} q\left(G_{i}\right)$.

3. $\left\lceil\frac{n-1}{2}\right\rceil \leq \operatorname{diam}\left(R_{v}\left(G_{1}, G_{2}, \ldots, G_{n}\right)\right) \leq\left\lceil\frac{\sum_{i=1}^{n} \operatorname{diam}\left(G_{i}\right)}{2}\right\rceil$.

The equality of both bounds are satisfied at complete graphs but the upper bound is satisfied at path graphs in which $v_{i}, u_{i}$ are end-vertices of $G_{i}$ for $i=1,2, \ldots, n$.

If $G_{i} \equiv H_{p}$, for all $1 \leq i \leq n$, where $H_{p}$ is a connected graph of order $p$, we denoted $R_{v}\left(H_{p}, H_{p}, \ldots, H_{p}\right)$ by $R_{v}\left(H_{p}\right)_{n}$.

Schultz and modified Schultz of $R_{v}\left(C_{6}\right)_{p / 2}$ :

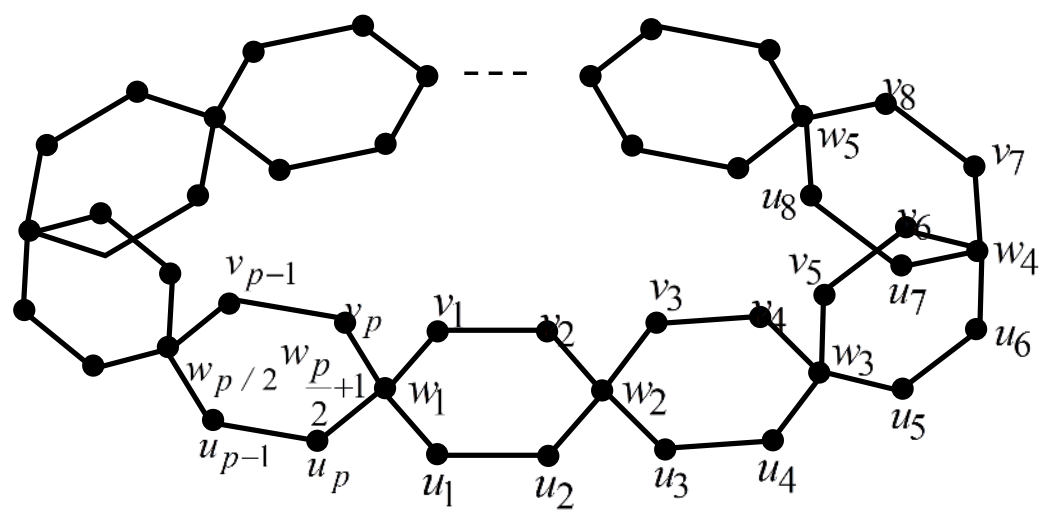

Fig. 2-2-2. The Graph $R_{v}\left(C_{6}\right)_{p / 2}, p \geq 6$, even $p$. 
From Fig. 2-2-2, we note that $\quad p\left(R_{v}\left(C_{6}\right) \frac{p}{2}\right)=\frac{5 p}{2}, q\left(R_{v}\left(C_{6}\right) \frac{p}{2}\right)=3 p \quad$ and $\operatorname{diam}\left(R_{v}\left(C_{6}\right) \frac{p}{2}\right)=\frac{p}{2}+\left\lceil\frac{p-2}{4}\right\rceil$. For all $1 \leq i, j \leq p, i \neq j$, then we have:

Table 2.1.1

\begin{tabular}{|c|c|c|c|}
\hline & $\operatorname{deg} u_{i}=2$ & $\operatorname{deg} v_{i}=2$ & $\operatorname{deg} w_{i}=4$ \\
\hline $\operatorname{deg} u_{j}=2$ & $\frac{4}{4}$ & 4 & $\frac{6}{8}$ \\
\hline $\operatorname{deg} v_{j}=2$ & $\frac{4}{4}$ & & $\frac{6}{8}$ \\
\hline $\operatorname{deg} w_{j}=4$ & $\frac{6}{8}$ & $\frac{6}{8}$ & $\frac{8}{16}$ \\
\hline
\end{tabular}

Theorem 2.1.2: For $p \geq 8$, then we have:

1. $S c\left(R_{v}\left(C_{6}\right) \frac{p}{2} ; x\right)=16 p x+24 p x^{2}+24 p x^{3}$

$$
+\left\{\begin{array}{c}
20 p \sum_{k=4,5,6, \ldots}^{\frac{p}{2}+\left[\frac{p-2}{4}\right]-1} x^{k}+10 p x^{\frac{p}{2}+\left[\frac{p-2}{4}\right\rceil}, \text { when } p=12,16,20, \ldots \\
20 p \sum_{k=4,5,6, \ldots}^{\frac{p}{2}+\left[\frac{p-2}{4}\right\rceil} x^{k}, \text { when } p=14,18,22, \ldots
\end{array}\right.
$$

2. $S c^{*}\left(R_{v}\left(C_{6}\right) \frac{p}{2} ; x\right)=20 p x+28 p x^{2}+28 p x^{3}$

$$
+\left\{\begin{array}{c}
24 p \sum_{k=4,5,6, \ldots}^{\frac{p}{2}+\left[\frac{p-2}{4}\right]-1} x^{k}+12 p x^{\frac{p}{2}+\left[\frac{p-2}{4}\right], \text { when } p=12,16,20, \ldots} \\
24 p \sum_{k=4,5,6, \ldots}^{\frac{p}{2}+\left[\frac{p-2}{4}\right]} x^{k}, \text { when } p=14,18,22, \ldots
\end{array}\right.
$$

Proof: For all $p \geq 12$, and every two vertices $u, v \in V\left(R_{v}\left(C_{6}\right) \frac{p}{2}\right)$, there is $d(u, v)=k$, $1 \leq k \leq \frac{3 p}{2}$, we will have seven partitions for proof:

P1. If $d(u, v)=1$, then $\left|D_{1}\right|=3 p=q\left(R_{v}\left(C_{6}\right) \frac{p}{2}\right)$ and we have two subsets of the edge set:

P1.1 $\left|D_{1}(2,2)\right|=\left|\left\{\left(u_{2 i-1}, u_{2 i}\right),\left(v_{2 i-1}, v_{2 i}\right): 1 \leq i \leq \frac{p}{2}\right\}\right|=p$.

P1.2 $\left|D_{1}(2,4)\right|=\left|\left\{\left(u_{2 i-1}, w_{i}\right),\left(v_{2 i-1}, w_{i}\right),\left(u_{2 i}, w_{i+1}\right),\left(v_{2 i}, w_{i+1}\right): 1 \leq i \leq \frac{p}{2}\right\}\right|=2 p$, where $w_{\frac{p}{2}+1} \equiv w_{1}$.

P2. If $d(u, v)=2$, then, we have two subsets of $D_{2}$ :

P2.1 $\left|D_{2}(2,2)\right|=\mid\left\{\left(u_{2 i}, u_{2 i+1}\right),\left(v_{2 i}, v_{2 i+1}\right),\left(u_{2 i+1}, v_{2 i}\right),\left(v_{2 i+1}, u_{2 i}\right): 1 \leq i \leq \frac{p}{2}\right\} \cup$ $\left\{\left(u_{i}, v_{i}\right): 1 \leq i \leq p\right\} \mid=3 p$, where $u_{p+1} \equiv u_{1}$ and $v_{p+1} \equiv v_{1}$.

P2.2 $\left|D_{2}(2,4)\right|=\left|\left\{\left(u_{2 i-1}, w_{i+1}\right),\left(v_{2 i-1}, w_{i+1}\right),\left(u_{2 i}, w_{i}\right),\left(v_{2 i}, w_{i}\right): 1 \leq i \leq \frac{p}{2}\right\}\right|=2 p$, where $w_{p+1} \equiv w_{1}$.

Thus $\left|D_{2}\right|=5 p$.

P3. If $d(u, v)=3$, then,we have three subsets of $D_{3}$ :

P3.1 $\left|D_{3}(2,2)\right|=\mid\left\{\left(u_{i}, u_{i+2}\right),\left(v_{i}, v_{i+2}\right),\left(u_{i}, v_{i+2}\right),\left(v_{i}, u_{i+2}\right): 1 \leq i \leq p\right\} \cup$ 


$$
\left\{\left(u_{2 i}, v_{2 i-1}\right),\left(v_{2 i}, u_{2 i-1}\right): 1 \leq i \leq \frac{p}{2}\right\} \mid=5 p,
$$

where $u_{p+a} \equiv u_{a}$ and $v_{p+a} \equiv v_{a}, a=1,2$.

P3.2 $\left|D_{3}(4,4)\right|=\left|\left\{\left(w_{i}, w_{i+1}\right): 1 \leq i \leq \frac{p}{2}\right\}\right|=\frac{p}{2}$, where $w_{\frac{p}{2}+1} \equiv w_{1}$.

Thus $\left|D_{3}\right|=\frac{11 p}{2}$.

P4. If $d(u, v)=k$, when $k=3 j+4$, and $p=12,16,20, \ldots, \mathrm{j}=0,1,2, \ldots, \frac{p}{4}-2$, and when $p=14,18,22, \ldots, \mathrm{j}=0,1, \ldots, \frac{p-2}{4}-2$, then, we have two subsets of such $(u, v)$ pairs of $D_{k}$ :

P4.1 $\left|D_{k}(2,2)\right|=\mid\left\{\left(u_{2 i-1}, u_{2 i+\frac{2(k-1)}{3}}\right),\left(v_{2 i-1}, v_{2 i+\frac{2(k-1)}{3}}\right),\left(u_{2 i-1}, v_{2 i+\frac{2(k-1)}{3}}\right)\right.$,

$$
\left.\left(v_{2 i-1}, u_{2 i+\frac{2(k-1)}{3}}\right): 1 \leq i \leq \frac{p}{2}\right\} \mid=2 p
$$

where $u_{p+a} \equiv u_{a}$ and $v_{p+a} \equiv v_{a}, a=2,4,6, \ldots, \frac{2(k-1)}{3}$

P4.2 $\left|D_{k}(2,4)\right|=\mid\left\{\left(u_{2 i}, w_{i+\frac{k+2}{3}}\right),\left(v_{2 i}, w_{i+\frac{k+2}{3}}\right),\left(u_{2 i+\frac{2 k-5}{3}}, w_{i}\right),\left(v_{2 i+\frac{2 k-5}{3}}, w_{i}\right)\right.$ :

$\left.1 \leq i \leq \frac{p}{2}\right\} \mid=2 p$, where $u_{p+a} \equiv u_{a}$ and $v_{p+a} \equiv v_{a}, a=1,2,3, \ldots, \frac{2 k-5}{3}$, where $w_{\frac{p}{2}+b} \equiv w_{b}, b=1,2,3, \ldots, \frac{k+2}{3}$.

Thus $\left|D_{k}\right|=4 p, k=3 j+4$,for $\mathrm{j}=0,1,2, \ldots, \frac{p}{4}-2$.

P5. If $d(u, v)=k$, when $k=3 j+5$, and $p=12,16,20, \ldots, \mathrm{j}=0,1,2, \ldots, \frac{p}{4}-2$, and when $p=14,18,22, \ldots, \mathrm{j}=0,1,2, \ldots, \frac{p-2}{2}-2$, then, we have two subsets of such $(u, v)$ pairs of $D_{k}$ :

$$
\begin{gathered}
\text { P5.1 }\left|D_{k}(2,2)\right|=\mid\left\{\left(u_{2 i}, u_{2 i+\frac{2 k-1}{3}}\right),\left(v_{2 i}, v_{2 i+\frac{2 k-1}{3}}\right),\left(u_{2 i}, v_{2 i+\frac{2 k-1}{3}}\right),\left(v_{2 i}, u_{2 i+\frac{2 k-1}{3}}\right):\right. \\
\left.1 \leq i \leq \frac{p}{2}\right\} \mid=2 p,
\end{gathered}
$$

where $u_{p+a} \equiv u_{a}$ and $v_{p+a} \equiv v_{a}, a=1,2,3, \ldots, \frac{2 k-1}{3}$.

P5.2 $\left|D_{k}(2,4)\right|=\mid\left\{\left(u_{2 i-1}, w_{i+\frac{k+1}{3}}\right),\left(v_{2 i-1}, w_{i+\frac{k+1}{3}}\right),\left(u_{2 i+\frac{2(k-2)}{3}}, w_{i}\right),\left(v_{2 i+\frac{2(k-2)}{3}}, w_{i}\right)\right.$ :

$$
\left.1 \leq i \leq \frac{p}{2}\right\} \mid=2 p \text {, }
$$

where $u_{p+a} \equiv u_{a}, v_{p+a} \equiv v_{a}, a=2,4,6, \ldots, \frac{2(k-2)}{3}$ and $w_{\frac{p}{2}+b} \equiv w_{b}, b=1,2,3, \ldots, \frac{k+1}{3}$.

Thus $\left|D_{k}\right|=4 p, k=3 j+5$, for $\mathrm{j}=0,1,2, \ldots, \frac{p-2}{2}-2$.

P6. If $d(u, v)=k$, when $k=3 j+6$, and when $p=12,16,20, \ldots, \mathrm{j}=0,1,2, \ldots, \frac{p}{4}-3$, and when $p=14,18,22, \ldots, \mathrm{j}=0,1, \ldots, \frac{p-2}{4}-2$, then, we have three subsets of such $(u, v)$ pairs of $D_{k}$ :

P6.1 $\left|D_{k}(2,2)\right|=\left|\left\{\left(u_{i}, u_{i+\frac{2 k}{3}}\right),\left(v_{i}, v_{i+\frac{2 k}{3}}\right),\left(u_{i}, v_{i+\frac{2 k}{3}}\right),\left(v_{i}, u_{i+\frac{2 k}{3}}\right): 1 \leq i \leq p\right\}\right|=4 p$, where $u_{p+a} \equiv u_{a}$ and $v_{p+a} \equiv v_{a} a=1,2,3, \ldots, \frac{2 k}{3}$.

P6.2 $\left|D_{k}(4,4)\right|=\left|\left\{\left(w_{i}, w_{i+\frac{k}{3}}\right): 1 \leq i \leq \frac{p}{2}\right\}\right|=\frac{p}{2}$,

where $w_{\frac{p}{2}+b} \equiv w_{b}, b=1,2,3, \ldots, \frac{2(2 k-3)}{3}$.

Thus $\left|D_{k}\right|=\frac{9 p}{2}, k=3 j+6$,for $\mathrm{j}=0,1,2, \ldots, \frac{p}{4}-3$. 
P7. If $d(u, v)=\frac{p}{2}+\left\lceil\frac{p-2}{4}\right\rceil$, then we have:

a- If $p=12,16,20, \ldots$, then, we have two subsets of $D_{\frac{p}{2}+}\left[\frac{p-2}{4}\right]$ :

P7.1 $\left|D_{\frac{p}{2}+\left\lceil\frac{p-2}{4} \mid\right.}(2,2)\right|=\left|\left\{\left(u_{i}, u_{i+\frac{p}{2}}\right),\left(v_{i}, v_{i+\frac{p}{2}}\right),\left(u_{i}, v_{i+\frac{p}{2}}\right),\left(v_{i}, u_{i+\frac{p}{2}}\right): 1 \leq i \leq \frac{p}{2}\right\}\right|=2 p$.

P7.2 $\left|D_{\left.\frac{p}{2}+\mid \frac{p-2}{4}\right]}(4,4)\right|=\left|\left\{\left(w_{i}, w_{i+\frac{p}{4}}\right): 1 \leq i \leq \frac{p}{4}\right\}\right|=\frac{p}{4}$.

Thus $\left|D_{\frac{p}{2}+\left[\frac{p-2}{4} \mid\right.}\right|=\frac{9}{4} p$, for even $\frac{p}{2}$.

b- If $p=14,18,22, \ldots$ then , we have two subsets of $D_{\frac{p}{2}+}\left[\frac{p-2}{4}\right]$ :

Ṕ7.1 $\left|D_{\left.\frac{p}{2}+\mid \frac{p-2}{4}\right\rceil}(2,2)\right|=\left|\left\{\left(u_{i}, u_{i+\frac{p}{2}}\right),\left(v_{i}, v_{i+\frac{p}{2}}\right),\left(u_{i}, v_{i+\frac{p}{2}}\right),\left(v_{i}, u_{i+\frac{p}{2}}\right): 1 \leq i \leq \frac{p}{2}\right\}\right|$ $=2 p$.

Ṕ7.2 $\left|D_{\frac{p}{2}+\left[\frac{p-2}{4} \mid\right.}(2,4)\right|=\mid\left\{\left(u_{2 i}, w_{i+\frac{p+2}{4}}\right),\left(u_{2 i-1}, w_{i+\frac{p+2}{4}}\right),\left(v_{2 i}, w_{i+\frac{p+2}{4}}\right),\left(v_{2 i-1}, w_{i+\frac{p+2}{4}}\right):\right.$ $\left.1 \leq i \leq \frac{p}{2}\right\} \mid=2 p$,

where $w_{\frac{p}{2}+b} \equiv w_{b}, b=1,2,3, \ldots, \frac{p+2}{4}$.

Thus $\left|D_{\frac{p}{2}+\left[\frac{p-2}{4}\right]}\right|=4 p$, for odd $\frac{p}{2}$.

From $\mathrm{P}_{1}$ to $\mathrm{P}_{7}$ and Table 2.1.2, we have:

$$
\begin{aligned}
& S c\left(R_{e}\left(C_{6}\right) \frac{p}{2} ; x\right)=\{4(p)+6(2 p)\} x+\{4(3 p)+6(2 p-4)\} x^{2}+\left\{4(5 p)+8\left(\frac{p}{2}\right)\right\} x^{3} \\
& \left(\sum_{k=4,7,10, \ldots}^{\frac{p}{2}+\left[\frac{p-2}{4}\right]-2}\{4(2 p)+6(2 p)\} x^{k}+\sum_{k=5,8,11, \ldots}^{\frac{p}{2}+\left[\frac{p-2}{4}\right]-1}\{4(2 p)+6(2 p)\} x^{k}\right. \\
& \begin{array}{c}
+\sum_{k=6,9,12, \ldots}^{\frac{3 p}{2}-3}\left\{4(4 p)+8\left(\frac{p}{2}\right)\right\} x^{k}+\left\{4(2 p)+8\left(\frac{p}{4}\right)\right\} x^{\frac{p}{2}+\left[\frac{p-2}{4}\right]} \\
\text { when } p=12,16,20
\end{array} \\
& +\left\{\begin{array}{c}
\text { when } p=12,16,20, \ldots \\
\sum_{k=4,7,10, \ldots}^{\frac{p}{2}+\left[\frac{p-2}{4}\right]-3}\{4(2 p)+6(2 p)\} x^{k}+\sum_{k=5,8,11, \ldots}^{\frac{p}{2}+\left[\frac{p-2}{4}\right]-2}\{4(2 p)+6(2 p)\} x^{k}
\end{array}\right. \\
& \begin{array}{c}
+\sum_{k=6,9,12, \ldots .}^{\frac{3 p}{2}-1}\left\{4(4 p)+8\left(\frac{p}{2}\right)\right\} x^{k}+\{4(2 p)+6(2 p)\} x^{\frac{p}{2}+\left\lceil\frac{p-2}{4}\right\rceil} \\
\text { when } p=14,18,22, \ldots
\end{array} \\
& =16 p x+24 p x^{2}+24 p x^{3} \\
& \left(20 p \sum_{k=4,7,10, \ldots}^{\frac{p}{2}+\left[\frac{p-2}{4}\right]-2} x^{k}+20 p \sum_{k=5,8,11, \ldots}^{\frac{p}{2}+\left[\frac{p-2}{4}\right]-1} x^{k}+20 p \sum_{k=6,9,12, \ldots}^{\frac{3 p}{2}-3} x^{k}\right.
\end{aligned}
$$

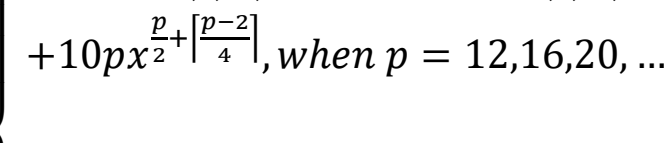

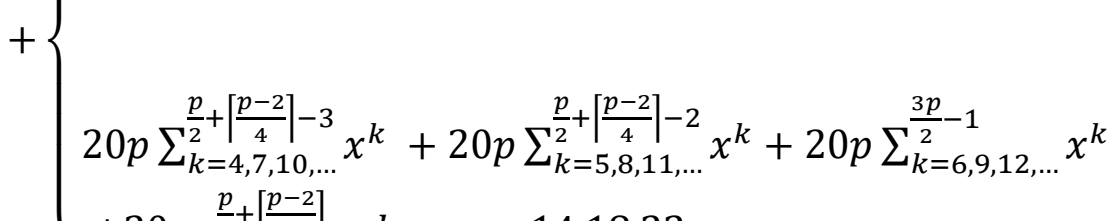

$$
\begin{aligned}
& +20 p x^{\frac{p}{2}+\left[\frac{p-2}{4}\right]}, \text { when } p=14,18,22, \ldots \\
& =16 p x+24 p x^{2}+24 p x^{3} \\
& +\left\{\begin{array}{l}
20 p \sum_{k=4}^{\frac{p}{2}+\left[\frac{p-2}{4}\right]-1} x^{k}+10 p x^{\frac{p}{2}+\left[\frac{p-2}{4}\right], \text { when } p=12,16,20, \ldots} \\
20 p \sum_{k=4}^{\frac{p}{2}+\left[\frac{p-2}{4}\right]} x^{k}, \text { when } p=14,18,22, \ldots
\end{array}\right.
\end{aligned}
$$

Now, we find modified Shultz polynomial: 


$$
\begin{aligned}
& S c^{*}\left(R_{v}\left(C_{6}\right) \frac{p}{2} ; x\right)=\{4(p)+8(2 p)\} x+\{4(3 p)+8(2 p-4)\} x^{2}+\left\{4(5 p)+16\left(\frac{p}{2}\right)\right\} x^{3} \\
& \left(\sum_{k=4,7,10, \ldots}^{\frac{p}{2}+\left[\frac{p-2}{4}\right]-2}\{4(2 p)+8(2 p)\} x^{k}+\sum_{k=5,8,11, \ldots}^{\frac{p}{2}+\left[\frac{p-2}{4}\right]-1}\{4(2 p)+8(2 p)\} x^{k}\right. \\
& +\sum_{k=6,9,12, \ldots}^{\frac{3 p}{2}-3}\left\{4(4 p)+16\left(\frac{p}{2}\right)\right\} x^{k}+\left\{4(2 p)+16\left(\frac{p}{4}\right)\right\} x^{\frac{p}{2}+\left\lceil\frac{p-2}{4}\right\rceil} \\
& +\left\{\begin{array}{c}
\text { when } p=12,16,20, \ldots \\
\sum_{k=4,7,10, \ldots}^{\frac{p}{2}+\left[\frac{p-2}{4}\right]-3}\{4(2 p)+8(2 p)\} x^{k}+\sum_{k=5,8,11, \ldots}^{\frac{p}{2}+\left[\frac{p-2}{4}\right]-2}\{4(2 p)+8(2 p)\} x^{k}
\end{array}\right. \\
& \begin{array}{c}
+\sum_{k=6,9,12, \ldots .}^{\frac{3 p}{2}-1}\left\{4(4 p)+16\left(\frac{p}{2}\right)\right\} x^{k}+\{4(2 p)+8(2 p)\} x^{\left.\frac{p}{2}+\mid \frac{p-2}{4}\right\rceil} \\
\text { when } p=14,18,22, \ldots
\end{array} \\
& =20 p x+28 p x^{2}+28 p x^{3} \\
& \left(24 p \sum_{k=4,7,10, \ldots}^{\frac{p}{2}+\left[\frac{p-2}{4}\right]-2} x^{k}+24 p \sum_{k=5,8,11, \ldots}^{\frac{p}{2}+\left[\frac{p-2}{4}\right]-1} x^{k}+24 p \sum_{k=6,9,12, \ldots}^{\frac{3 p}{2}-3} x^{k}\right. \\
& +12 p x^{\frac{p}{2}+\left[\frac{p-2}{4}\right]}, \text { when } p=12,16,20, \ldots \\
& +\left\{\begin{array}{l}
24 p \sum_{k=4,7,10, \ldots}^{\frac{p}{2}+\left[\frac{p-2}{4}\right]-3} x^{k}+24 p \sum_{k=5,8,11, \ldots}^{\frac{p}{2}+\left[\frac{p-2}{4}\right]-2} x^{k}+24 p \sum_{k=6,9,12, \ldots}^{\frac{3 p}{2}-1} x^{k}
\end{array}\right. \\
& +24 p x^{\frac{p}{2}+\left\lceil\frac{p-2}{4}\right\rceil}, \text { when } p=14,18,22, \ldots \\
& =20 p x+28 p x^{2}+28 p x^{3} \\
& +\left\{\begin{array}{l}
24 p \sum_{k=4}^{\frac{p}{2}+\left[\frac{p-2}{4}\right]-1} x^{k}+12 p x^{\frac{p}{2}+\left[\frac{p-2}{4}\right]}, \text { when } p=12,16,20, \ldots \\
24 p \sum_{k=4}^{\frac{p}{2}+\left[\frac{p-2}{4}\right]} x^{k}, \text { when } p=14,18,22, \ldots
\end{array}\right.
\end{aligned}
$$

By simply, we can calculate:
1. $S c\left(R_{v}\left(C_{6}\right)_{4} ; x\right)=128 x+192 x^{2}+192 x^{3}+160 x^{4}+160 x^{5}+80 x^{6}$. $S c^{*}\left(R_{v}\left(C_{6}\right)_{4} ; x\right)=160 x+224 x^{2}+224 x^{3}+192 x^{4}+192 x^{5}+96 x^{6}$.
2. $S c\left(R_{v}\left(C_{6}\right)_{5} ; x\right)=160 x+240 x^{2}+240 x^{3}+200 x^{4}+200 x^{5}+200 x^{6}+200 x^{7}$. $S c^{*}\left(R_{v}\left(C_{6}\right)_{5} ; x\right)=200 x+280 x^{2}+280 x^{3}+240 x^{4}+240 x^{5}+240 x^{6}+240 x^{7}$.

\section{Remark:}

1. $S c\left(R_{v}\left(C_{6}\right)_{2} ; x\right)=64 x+96 x^{2}+56 x^{3}$. $S c^{*}\left(R_{v}\left(C_{6}\right)_{2} ; x\right)=80 x+112 x^{2}+64 x^{3}$.

2. $S c\left(R_{v}\left(C_{6}\right)_{3} ; x\right)=96 x+144 x^{2}+144 x^{3}+120 x^{4}$. $S c^{*}\left(R_{v}\left(C_{6}\right)_{3} ; x\right)=120 x+168 x^{2}+168 x^{3}+144 x^{4}$.

Corollary 2.1.2: For $p \geq 4$, then we have:
1. $S c\left(R_{v}\left(C_{6}\right) \frac{p}{2}\right)=\left\{\begin{array}{l}\frac{p}{8}\left(45 p^{2}+128\right), \text { when } p=4,8,12, \ldots \\ \frac{3 p}{8}\left(15 p^{2}+36\right), \text { when } p=6,10,14 \ldots\end{array}\right.$
2. $S c^{*}\left(R_{v}\left(C_{6}\right) \frac{p}{2}\right)=\left\{\begin{array}{l}\frac{p}{4}\left(27 p^{2}+64\right), \text { when } p=4,8,12, \ldots \\ \frac{p}{4}\left(27 p^{2}+52\right), \text { when } p=6,10,14, \ldots\end{array}\right.$.

Corollary 2.1.3: If $n$ is the number of cycles $C_{6}$ in the graph $R_{v}\left(C_{6}\right)_{n}, n \geq 2$, then we have: 
1. $S c\left(R_{v}\left(C_{6}\right)_{n}\right)=\left\{\begin{array}{l}n\left(45 n^{2}+32\right), \text { when } n=2,4,6, \ldots \\ 9 n\left(5 n^{2}+3\right), \text { when } n=3,5,7, \ldots\end{array}\right.$

2. $S c^{*}\left(R_{v}\left(C_{6}\right)_{n}\right)=\left\{\begin{array}{l}2 n\left(27 n^{2}+16\right), \text { when } n=2,4,6, \ldots \\ 2 n\left(27 n^{2}+13\right), \text { when } n=3,5,7, \ldots\end{array}\right.$

Corollary 2.1.4: For $p \geq 4$, then we have:

1. $\overline{S c}\left(R_{v}\left(C_{6}\right) \frac{p}{2}\right)=\left\{\begin{array}{l}\frac{1}{5}\left(9 p+\frac{18}{5}+\frac{676}{5(5 p-2)}\right), \text { when } p=4,8,12, \ldots \\ \frac{3}{5}\left(3 p+\frac{6}{5}+\frac{162}{5(5 p-2)}\right), \text { when } p=6,10,14, \ldots\end{array}\right.$

2. $\overline{S c^{*}}\left(C_{v}\left(C_{6}\right) \frac{p}{2}\right)=\left\{\begin{array}{l}\frac{2}{5}\left(27 p+\frac{54}{5}+\frac{1708}{5(5 p-2)}\right), \text { when } p=4,8,12, \ldots \\ \frac{2}{25}\left(27 p+\frac{54}{5}+\frac{1408}{5(5 p-2)}\right), \text { when } p=6,10,14, \ldots\end{array}\right.$. 


\section{REFERENCES}

[1] Ahmed M.A., Haitham N.M., (2017); "Schultz and Modified Schultz Polynomials of two Operations Gutman's", International Journal of Enhanced Research in Science, Technology \& Engineering. 6, pp.68-74.

[2] Ahmed M.A. and Haitham N.M.; (2019), Schultz and Modified Schultz Polynomials of Some Cog-Special Graphs, Open Access Library Journal, Vol.6, pp.1-13.

[3] Behmaram, A., Yousefi-Azari, H. and Ashrafi, A.R., (2011); "Some New Results on Distance - Based Polynomials", MATH. Commun. Math. Comput. Chem. 65, pp.39-50.

[4] Buckley, F. and Harary, F.; (1990); Distance in Graphs, Addison - Wesley, Longman.

[5] Chartrand, G. and Lesniak, L.; (2016); Graphs and Digraphs, $6^{\text {th }}$ ed., Wadsworth and Brooks / Cole. California.

[6] Farahaini M. R., (2013); "Hosoya, Schultz Modified Schultz Polynomials and their Topological Indices of Benzene Molecules: First Members of polycyclic Aromatic Hydro Carbons (PAHs)"; International Journal of theoretical chemistry Vol. 1, No. 2, pp. 6-9.

[7] Farahaini M.R., (2014); "Schultz and Modified Schultz Polynomials of Coronene Polycyclic Aromatic Hydro carbons", International Letters of chemistry, Physics and Astronomy, Vol. 32, pp. 1-10.

[8] Farahaini M.R.; (2013); On the Schultz Polynomial, Modified Schultz Polynomial, Hosoya polynomial and Wiener Index of Circumcoronene Series of Benzenoid. J. Appl. Math. \& Informatics Vol. 31, No. 5 - 6, pp. 595 - 608.

[9] Farahani M.R., (2013); "On the Schultz and Modified Schultz Polynomials of Some Harary Graphs", International Journal of Applications of Discrete Mathematics; Vol. 1, No. 1, pp. 01- 08.

[10] Farahani M.R., Wang, S., Wei, G., BING WEI4 and Jamil, M.K.; (2018); "The Hosoya Schultz and Modified Schultz of Class od Dutch Windmill Graph $D_{n}^{(m)}$, $\forall \mathrm{n}, \mathrm{m} \in \mathrm{N} \& \mathrm{n} \geq 4, \mathrm{~m} \geq 2$ ". Communications in Applied Analysis, 22, No. 1, pp. 43-62.

[11] Guo. H. and Zhow B.; (2017); "Properties of Degree Distance and Gutman Index of Uniform Hypergraphs" MATCH Commun. Math. Comput Chem. 78, pp. 213-220.

[12] Gutman, I. (2005), "Some Relations Between Distance- Based Polynomials of Trees”. Bull. A cod. Serbe. Sci. Arts 131, pp.1-7.

[13] Haneen, K.A.; (2017); Schultz index, Modified Schultz index, Schultz polynomial and Modified Schultz polynomial of alkanes. Global Journal of Pure and Applied Mathematics. Vol. 13, No. 9, pp. 5827-5850.

[14] Hassani, G., Iranmanesh, A. and Mirzaie, S. (2013), "Schultz and Modified Schultz Polynomials of C100 Fullerene". MATCH Commun. Math. Comput. Chem. Vol. 69, pp. 87-92. 
[15] Heydari, A., (2010), "On the Modified Schultz Index of C4C8(S) Nanotubes and Nanotours. Digest Journal of Nanomatrial and Biostructures, 5, pp. 51-56.

[16] Iranmanesh, A. and Ali zadeh, Y., (2009); Computing Szeged and Schultz Indices of HAC3C7C9 [p,q] Nanotube by Gap program. Digest Biostructures, 4, pp. 67-72.

[17] Klavžar, S. and Gutman, I., (1997); Wiener number of vertex-weighted graphs and a chemical application. Disc. Appl. Math. 80, pp.73-81.

[18] Schultz, H.P., (1989); Topological organic chemistry 1. Graph theory and topological indices of alkanes. J. Chem. Inf. Comput. Sci. 29, pp.227-228. 